\title{
Internet of Things: Visions, Technologies, and Areas of Application
}

\author{
Amine Rghioui, Abedlmajid Oumnad \\ Electronic and Communication Laboratory-LEC, EMI, Mohammed V University, Rabat, Morocco
}

\section{Email address:}

rghioui.amine@gmail.com (A. Rghioui),aoumnad@emi.ac.ma (A. Oumnad)

\section{To cite this article:}

Amine Rghioui, Abedlmajid Oumnad. Internet of Things: Visions, Technologies, and Areas of Application. Automation, Control and Intelligent Systems. Vol. 5, No. 6, 2017, pp. 83-91. doi: 10.11648/j.acis.20170506.11

Received: September 11, 2017; Accepted: October 23, 2017; Published: November 29, 2017

\begin{abstract}
The internet of things (IoT), also called internet of all, is a new paradigm that combines several technologies such as computers, the internet, sensors network, radio frequency identification (RFID), communication technology and embedded systems to form a system that links the real worlds with digital worlds. IoT is recognized as one of the most important areas of the technology of the future and wins attention from a wide range of industries. Currently, a large number of smart objects and different types of devices are interconnected and communicate using the internet protocol. With an increase in the deployment of smart objects, the internet of things should have a significant impact on human life in the near future. To understand the development of the IoT, this paper reviews the current research of the IoT, key technologies, the main applications of the IoT in various field, and identifies research challenges. In this article, we present the main visions and the scope of the internet of things and the futuristic research and its fields of application. the main contribution of this review article is that it summarizes the current state of the IoT technology in several areas, and also the applications of IoT that cause side effects on our environment for monitoring and evaluation of the impact of human activity on the environment around us, and also provided an overview of some of the main challenges of IoT, and also shows that application of the IoT. This article presents not only the problems and challenges of IoT, but also solutions that help overcome some of the problems and challenges.
\end{abstract}

Keywords: Internet of Things, Radio Frequency Identification, WSN, Cloud Computing, Embedded Systems, Smart Object

\section{Introduction}

This paper presents a review of literature on the subject of the internet of things technologies and their applications domains and the futuristic research areas. Several research studies have addressed and developed this topic with detailed studies synthesis about the fields of application of internet of things, and general visions $[1,2]$. Throughout this paper, we propose a state of the art on the technologies of the internet of things and their areas of application and current research in the areas of health, industry, robotics, transport and logistics (production, distribution, transportation, maintenance, marketing, management).

The Internet of Things (IoT) is a computing concept that describes a future where every day physical objects will be connected to the Internet and be able to identify themselves to other devices.

The term "internet of things" has emerged 20 years ago in downtown MIT auto-ID1 marked the beginning of a new era for trade and industry. First, the internet of things was regarded as a simple extension of the identification by radio frequency (RFID). But if you consider the possibilities of evolutions and the number of applications attached to the interconnection of objects, the internet of objects appears more as a revolution: during the $19^{\text {th }}$ century, machines have learned to execute commands during the $20^{\text {th }}$ century, they have learned to think, and in the $21^{\text {st }}$ century, they will learn to anticipate and perceive $[3,4]$.

Over the past three decades, a fantastic job on the internet has led to the growth of the internet of things where are created intelligent interconnections between various objects. The main vision behind the internet of things is that devices shipped, also known as smart objects, are increasingly organizing connected between them [5].

The technology of the internet of things (IoT) establishes a connection between all things and the Internet. IoT is widely applied in intelligent transportation, environmental protection, public safety, positioning, tracking, and 
monitoring and management intelligently. Intelligent building and house residential area based on the application of information technology little become more imperative.

In this article, we aim to provide a global perspective on the concept and development of internet of things, including a critical review of application domains, enabling technologies and challenges of research. In fact, the community of active research on topics related to the (IoT) is still very fragmented, and, largely, focused areas of simple applications or simple technologies. In addition, the participation of the communities for networking and scientific communication is still limited, despite the potential impact of their contributions on the development of the field $[6,7]$.

The technology of internet of things (IoT) establishes a connection between all things, and internet via detection of devices and smart tools identification and management. The means of remote sensing information includes RFID, infrared, GPS and laser sensor devices scanner. They are all connected to the internet to implement control and distance perception [8]. IoT is widely applied in intelligent transportation, environmental protection, the governmental jobs, public safety smart homes, fire control, industrial monitoring, and care for the older persons, health personnel stuff, etc...

Several industrial entities, research and standardization are currently involved in the activity of developing solutions to meet the technological needs highlighted. This survey gives a picture of the current state of the art on the IoT. More specifically, it: provide readers a description of the different visions of the internet of things paradigm from different scientific communities. The main objective is to give the reader the opportunity to understand what has been done and what remains to be done in this area.

The rest of this article is organized as follows. In Section 2, we showing us and explaining other surveys on the Internet of Things. In section 3 , we introduce the essential IoT technologies. In section 4 , we give a general overview of the challenges of the Internet of Things. Application fields of Internet of Things are defined in section 5. Section 6 is devoted to describing and present IoT futuristic applications Section 7 concludes the investigation with a number of remarks on possible approaches.

\section{Other Surveys on IoT}

In this part, we showed the main research areas considered by most surveys published in the field of the Internet of Things. To clarify that the IoT refers to several good surveys recently presented each display IoT from a different perspective: challenges [3] applications [8] and standards [6]. Among these investigations, a complete overview of IoT, and three different angles: things, Internet, and semantics, was presented by Atzori and her colleagues [7]. There are several documents published survey covering different aspects of IoT technology. For example, the survey by Eleonora Borgia [9] covers the main communication enabling technologies, and the elements of wireless sensor networks (WSN). In addition, [12] discusses the IoT in terms of enabling technologies focusing on RFID and its potential applications. IoT challenges presented in [13] to bridge the gap between research and the practical aspects. An overview of the standards and challenges for current IoT presented in [14]. All that for IoT technologies and research challenges for the applications of the Internet of things, there are many surveys that address what this part of applications for example in [15] the authors come up with a solution to manage IoT bicycle parking very effectively is a parking for bicycles. A project for smart water monitoring and management of the water cycle was presented in [16]. A tracking application people and inventory logistics was presented in [17]. Another IoT application that is very important in the field of mining is mining production which is developed by [18] for Safety in mines, to prevent and reduce accidents in the mining sector, Using RFID, Wi-Fi and other wireless communication technologies. Another useful application is to use chemical and biological sensors for early detection of the disease and diagnosis of minors. The IoT use in the transport and logistics is given by [19]. Zhang et al. [20] developed an intelligent control system to monitor the temperature / humidity inside refrigerated trucks using RFID tags, sensors and wireless communication technology.

To the best of our knowledge, however, no investigation has focused on industrial solutions of IoT. All the above investigations have reviewed the solutions proposed by academic and research communities and to refer to scholarly publications produced by the respective researchers. In this article, we review the problems, challenges, technologies and IoT applications that are proposed, designed, developed and marketed, and are useful for researchers and industrial organizations.

This paper begins by providing a horizontal overview of the IoT. Next, we give an overview of some technical details that are relevant to the IoT enabling technologies. Compared to other documents from the field survey, our goal is to provide a more detailed summary of IoT technologies, research challenges, problems, and existing applications to enable researchers and application developers to see that they are the areas covered by the Internet of Things.

The contour of the contributions of this paper compared to the recent literature in the field can summarized as follows:

i. Compared to other documents from the field survey, this survey provides a deeper summary of the Internet of Things, which allows us to know what the Internet of Things is in details.

ii. We provide an overview of some of the main challenges of IoT presented in recent literature and provide a summary of related research. In addition, we explore the relationship between the IoT and other emerging technologies: sensor networks, RFID technology, and cloud computing.

iii. We express the need for better horizontal integration between IoT services.

iv. We also present the different fields of application of the 
Internet of Things in the human life, and the detailed futuristic applications to illustrate the further work in this area.

\section{Internet of Things Technologies}

A novel paradigm called Internet of Things (IoT) has rapidly gained ground in recent years. IoT refers to "a global network of interconnected objects that are uniquely addressable based on standard communication protocols", the point of convergence of which is the Internet. IoT is powered by the latest advances in a variety of communication devices and technologies, but the things included in IoT are not just complex devices such as mobile phones, but they also include everyday objects such as food, clothing, furniture, paper, landmarks, monuments, works of art, etc.. These objects, acting as sensors or actuators, are capable of interacting with each other to achieve a common goal. In the following, we describe some very important technological aspects related to the IoT.

\subsection{RFID Technology (Radio Frequency Identification)}

RFID is an automatic and contactless technology, providing a communication interface with the tagged objects through wireless data transmission to retrieve relevant information. [21] Radio frequency identification (RFID) allows automatic identification and data capture using radio waves, a tag, and a reader. The tag cab store more data than traditional barcodes. Three types of tags are used.

Passive RFID tags rely on radio frequency energy transferred from the reader to the tag to power the tag; they are not battery-powered. Passive RFID technologies present many advantages. Indeed, RFID can be seen as "electronic bar codes" which do not need objects to be handled one by one. In addition, no direct sight is necessary. More and more information can be stored in tags. Tag reading is quick (a reader can read up to 250 tags per second) [22]. Applications of these can be found in supply chains, passports, and electronic tolls.

Active RFID tags can contain external sensors to monitor temperature, pressure, chemicals, and other conditions. Active RFID tags are used in manufacturing, hospital laboratories, and remote-sensing IT asset management.

Semi-passive RFID tags use batteries to power the microchip while communicating by drawing power from the reader. Active and semi-passive RFID tags cost more than passive tags.

In IoT scenario, a key role is played by RFID systems, composed of one or more readers and several tags. These technologies help in automatic identification of anything they are attached to, and allow objects to be assigned unique digital identities, to be integrated into a network, and to be associated with digital information and services.

\subsection{Wireless Sensor Networks (WSN)}

A Wireless Sensor Network (WSN) can be defined as a network of small embedded devices, called sensors, which communicate wirelessly following an ad hoc configuration. [23]. Wireless sensor networks (WSN) consist of spatially distributed autonomous sensor-equipped devices to monitor physical or environmental conditions and can cooperate with RFID systems to better track the status of things such as movements, pressure, temperature, and location. Wireless sensor networks (WSN) my provide various useful data and are being utilized in several areas like healthcare, government and environmental services (natural disaster relief), defense (military target tracking and surveillance), hazardous environment exploration, seismic sensing. (WSN are used for maintenance and tracking systems. For example, General Electric deployed sensors in its jet engines, turbines, and wind farms. By analyzing data in real time, General Electric saves time and money associated with preventive maintenance. Likewise, American Airlines uses sensors capable of capturing 30 terabytes of data per flight for services such as preventive maintenance.

\subsection{Middleware}

Middleware is a software layer interposed between software applications to make it easier for software developers to perform communication and input/ output. Middleware gained popularity in the 1980 due to its major role in simplifying the integration of legacy technologies into new ones. It also facilitated the development of new services in the distributed computing environment.

A complex distributed infrastructure of the IoT with numerous heterogeneous devices requires simplifying the development of new applications and services, so the use of middleware is an ideal fit with IoT application development. For example, Global Sensor Network (GSN) is an open source sensor middleware platform enabling the development and deployment of sensor services with almost zero programming effort.

Due to the heterogeneity of the participating objects, to their limited storage and processing capabilities and the huge variety of applications involved, a key role is played by the middleware between the things and the application layer, whose main goal is the abstraction of the functionalities and communication capabilities of the devices.

The middleware can be divided in a set of layers: Object Abstraction, Service Management, Service Composition, and Application. [24]

\subsection{Cloud Computing}

The essential aspects of cloud computing have been reported in the definition provided by the National Institute of Standard and Technologies (NIST): "Cloud computing is a model for on demand access to a shared pool of configurable resources (e.g., computers, networks, servers, storage, applications, services, software)" that can be provisioned as infrastructure as a service or software of the IoT is an enormous amount of data generated from devices connected to the internet. 
Many IoT applications require massive data storage, huge processing speed to enable real-time decision making, and high-speed broadband networks to stream data, audio, or video. Cloud computing provides an ideal back-end solution for handling huge data streams and processing them for the unprecedented number of IoT devices and humans in realtime. [25].

\section{Challenges of Internet of Things}

Security challenges can be addressed by training developers to integrate security solutions (such as firewall, firewall, etc.) into products and by encouraging users to use the security features of the IdO which are built into their devices

\subsection{Technology Challenges}

The Internet of Things (IdO) as a new global Internetbased information architecture that facilitates the exchange of goods and services are gradually gaining in importance.

The best-known use of the IdO is based on RFID (Radio Frequency Identification Device). In practice, the level of sophistication and RFID prices can be very different, starting with the cheap passive device without a power supply and storage limited to an active RFID having advanced storage and communication capabilities.

Some of the data collected seem to be insignificant, but for example, data relating to a production process could be very valuable, thus requiring appropriate protection.. In addition, all smart phones today carry position sensors by allowing them to continuously monitor their users. All these IdO devices in a form add value to individuals as well as businesses; However, they also cause risks.

\subsection{Security Challenges}

The IdO devices collect a large amount of information and therefore they carry a great potential for privacy risks as compared to using the data and accessing it. As more and more IoT devices used in all areas of everyday life, such as in the health care sector, a large amount of private information considered is stored and collected. [26]

As a growing number and variety of connected devices are introduced into IO networks, the potential threat to security degenerates. While IdO improves business productivity and improves the quality of people's lives, the IdO also increase potential attack surfaces for hackers and other cybercriminals. A recent study found that $70 \%$ of the more commonly used IdO devices contain severe vulnerabilities.

Application Fields of IoT

\begin{tabular}{|c|}
\hline $\begin{array}{c}\text { Transportation } \\
\text { and logistics }\end{array}$ \\
\hline Logistics \\
A $\begin{array}{c}\text { Assisted } \\
\text { driving } \\
\text { Mobile } \\
\text { ticketing } \\
\text { The monitoring } \\
\text { of products } \\
\text { transported }\end{array}$ \\
\hline
\end{tabular}
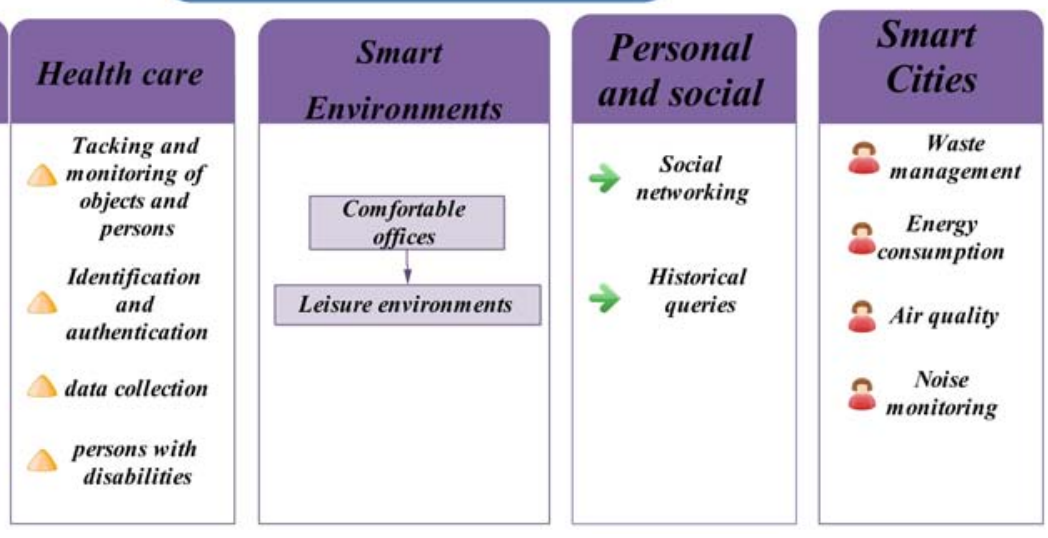

Figure 1. Fields of applications of the internet of things.

\section{Domain of Applications}

Applications can be grouped into six main areas: industrial domain, smart city, intelligent environments, social domain, and the domain of health. Each domain is not isolated from the others, but it is partially overlapping because some application are shared. An example is the tracking of products, which is in common between industrialists and the domain of health because they can be used for the monitoring of food, but it is also able to monitor the delivery of pharmaceuticals. Figure 1 shows the subdivision of the fields of application of internet of things.

\subsection{Transportation and Logistics}

The transport and logistics sector is a promising sector for the economy, the importance of the sector of transport and logistics is measured also by its direct impact on the competitiveness of the economic fabric as well in terms of export as import that is why one finds several application in this field such as logistics, helps conduct, mobile ticketing...etc.

\subsubsection{Logistics}

Applications of IoT in the logistics field include an application industrial is the management of the supply chain and procurement. RFID can be attached to an object and allows identification of materials and products, such as clothing, or food and liquids. Their use helps to effectively manage ware houses and simplify inventory by providing a precise knowledge of the current inventory, while reducing inventory inaccuracies. 


\subsubsection{Assisted Driving}

Cars, trains and buses as well as roads are equipped by sensors and actuators to provide important information to the driver or passenger of a car to allow better navigation and good security. Also there is monitoring of hazardous materials transportation systems. In addition, government authorities would benefit from these applications at the level to have more accurate information on road traffic patterns.

\subsubsection{Mobile Ticketing}

In this axis there are posters in railway stations or airports which provided information regarding transportation such as the prize time... these posters are equipped with tags NFC (Near Field Communication: is a standard based short-range wireless connection technology that enabled simple and safe peer-to-peer interconnections between electronics devices). The user can then obtain information on several categories of options on the web either by its hovering mobile phone on the NFC tag, or by pointing the mobile phone for visual markers. The mobile phone automatically gets information of the associated web services(stations, number of passengers, costs, availability and type of services) this application also allows the user to buy tickets online [27].

\subsubsection{The Monitoring of Products Transported}

Among the goods transported are fresh products such as fruit and meat also dairy, they are vital products of our foods. For the transported thousands of kilometers, it should be monitored to avoid the uncertainty of the level of the quality of the goods. Working on ubiquitous computing and sensor technologies, these technologies are able to maintain the temperature and humidity at the state normal.

\subsection{Health Care Domain}

The internet of things will play an essential role to develop intelligent services and to support and enhance the activities of the society and the people. These services enable peoples to live independently, and to improve their health for this there are many benefits offered by the internet of things technologies include: tracking objects and people (staff and patients), medical parameters monitoring and administration of the drugs, the identification and authentication of people, collects data automatic and remote sensing [28].

\subsubsection{Tacking and Monitoring of Objects and Persons}

Monitoring is the function aims at the identification of a person or an object in motion. In the field of health there are tracking and monitoring of patient flow to improve the workflow in hospitals and motion tracking through choke points, such as access to designated areas. The follow-up is more frequently applied instead of permanent inventory of monitoring (e.g. for maintenance, availability in case of need and use monitoring) and tracking of materials in order to avoid problems during surgery.

\subsubsection{Identification and Authentication}

The identification of the patients is to reduce malicious patient incidents (e.g. bad drug/dose/time/procedure), also the identification of the medical file complete and current, with regard to staff, the identification and authentication is most often used to grant access and improve the morale of the employees based on the safety of patients, are used as identification and authentication to meet the requirements of thee safety procedures to prevent the theft or loss of products and instruments.

\subsubsection{Transport and Data Collection}

Nowadays, personal health devices can transmit data using short-range Bluetooth wireless technologies, Near Field Communication (NFC), ZigBee or Bluetooth Low Energy (BLE), to mention some [29]. Transfer and automatic data collection is to reduce the time of processing forms, automated care and audit and management of medical procedures. This function also refers to integration RFID technology.

\subsubsection{Application for Persons with Disabilities}

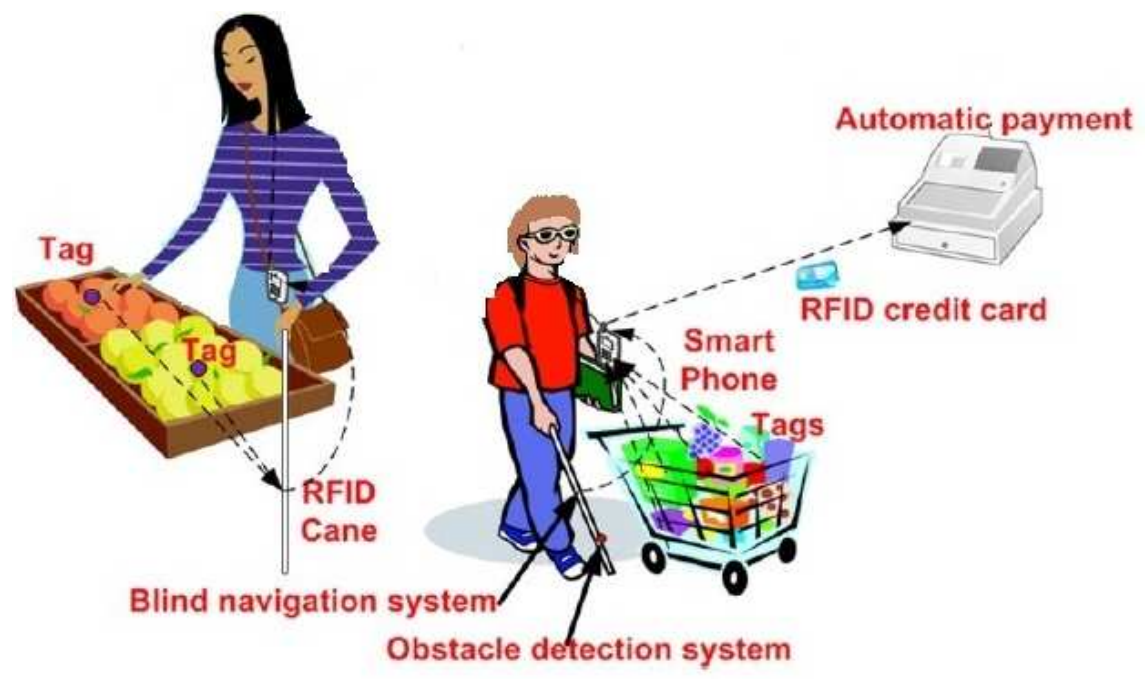

Figure 2. System of blind navigation for shopping. 
There is an application called system of blind navigation for people with visual impairments to shopping. This blind navigation system helps these people to find their way in a store. Base system RFID store can use software to guide the visually impaired in shopping as indicated in Figure 2 the supermarket is divided into cells containing one shelf and the passage cells. RFID tags are distributed through the floor. Monitoring station (smart phone) maintains a Bluetooth connection with the reader RFID (intelligent cane) of the user [30].

The internet of things will play a very important role of the field of health in the near future because application in this area are enormous and these are very important and have many advent disabilities in the social economic, political and cultural fire. These applications makes it easy for people have a disability to make their activities every day it increases their autonomy and their self-confidence.

\subsection{Smart environments Domain}

An intelligent environment domain is a field concerned by the easy and comfortable use with comfortable workplaces and offices and the intelligence of content objects, be it a house, an industrial establishment, or a leisure environment.

\subsubsection{Comfortable Offices}

Sensors and actuators in the offices can make our life more comfortable in several respects: rooms heating of office lighting can change according to the hour of the day, appropriate monitoring and alarm systems, and also energy can be saved by automatically switched off of electricity equipment when not required.

\subsubsection{Leisure Environments}

Intelligent environments of leisure, like the Museum and the gym are two intelligent environments of leisure, like the Museum and the gym are two example where the internet of things technologies are exploited. For example the Museum can expose different historical periods with widely divergent of the climatic conditions. The premises of the Museum automatically adjusts with the external conditions (temperature, humidity...). Another application is in the gym, or trainers can download the profile of the exercise in the training for each player machine, when the player takes the machine, it will be automatically recognized through the label RFID connected to the machine. Also health parameters are monitored during the workout.

\subsection{Personal and Social Domain}

By analogy with the services of social networks for human beings, the internet of things introduces the concept of the social relationships between the objects. The advantages are the possibility of giving the internet of objects that can be shaped as needed to ensure the airworthiness of the network [31]. In this area there are applications that enable the user to interact with others to maintain and build social relationship. Indeed, things can automatically trigger the transmission of messages to friends so that they know what we do or what we have done in the past, share a few things in common.

\subsubsection{Social Networking}

We can think of RFID tags that generate events on the people and places to give-up dates in real time to users in their social networks such as Twitter, which are then collected and uploaded in networking sites social. Application user interfaces display a stream of events that friends have previously set and users can control their friend lists so that what events are disclosed to what friends. The field of the internet of things does not escape the trend of internet of things social. In the future internet, the majority of the connections will established among humans, but among devices (things, more or less "intelligent").

\subsubsection{Historical Queries}

Found several applications in the field of historical queries among these implementation is cited: loss (creation of a search engine for things which helps to find items that we do not remember or were left) and flights (application can send a SMS to users stolen objects leave their seats without permission).

\subsection{Industrial Domain}

A number of industrial projects of the internet of things were conducted in such areas as agriculture, processing of industrial food, environmental monitoring and security surveillance. The internet of things will play a role more important in the transport and logistics industries such as physical objects are equipped with barcodes, RFID tags or sensors, transport and logistics companies can conduct surveillance in real-time to move physical objects of an origin to a destination through the supply chain including manufacturing, shipping, distribution and so on [32]. Intelligent environments also helps improve the automation in industrial installations with a massive deployment of labels it RFID related for production parts.

\subsection{Areas of Smart Cities}

The "Smart City", is designed to make better use of public resources, increasing the quality of services offered to citizens, while reducing the operational costs of public administration found applications in many different areas, such as home automation, industrial automation, medical aid, mobile health care, elderly assistance, intelligent management of energy and smart grids, the automobile traffic, management and others [33].

There are also other applications in this field such as:

1. The state of the building structures.

2. Waste management.

3. Air quality.

4. Noise monitoring.

5. Attack on the traffic.

6. Energy consumption of city.

7. Smart parking. 
8. Intelligent lighting.

9. Automation and cleanliness of public buildings.

\section{Futuristic Research Areas}

The internet of things is a vision that embraces and overcomes several technologies at the confluence of the nanotechnology, biotechnology, information technology and cognitive science. During the 7 to 10 years, the internet of things is likely to quickly develop and shape a new "information society" and "knowledge economy".

\subsection{The Aerospace and Aviation}

The internet of things will strengthen the security of products and services protecting them from counterfeiting. A problem facing aviation, 28 incidents 4 have caused in the United States by counterfeit components not conforming to the safety standards. These incidents could be avoided by introducing an "electronic pedigree" which will shape the life cycle of the critical elements of the aircraft, from their manufacture to their use. This is done by linking the RFID technology to a dynamic database. This database can be coupled with other elements of the aircrafts as different sensors (pressure, temperature...) and safety systems.

\subsection{Telecommunications}

Based on multiple existing and future technologies (GSM, UMTS, LTE, NFC, Bluetooth, Wi-Fi, GPS and sensors), internet of things will promote the development of new applications and new services. For example in the case of the NFC, we communique simply and securely with different objects by "scanning" them with a mobile phone that will transmit the data to a server. The interconnection of objects creates a vast network of data exchange that allows even to keep a medium of communication in the event of failure of the current telecommunications infrastructure [34]. In addition, the management of personal data by the SIM card offers increased security for authentication, the exchange of confidential data, or even the payment by mobile.

\subsection{The Intelligent Building and Instrumentation of the Buildings}

Among the economic sectors, the building sector is the largest energy consumer. Technology advanced the internet of objects in this sector can help to reduce the consumption of resources related to building (electricity, water) as well as to improve the level of satisfaction of humans inhabiting, or workers in office buildings or tenants of private houses [35]. Solutions such as smart meters are becoming increasingly popular for measuring energy consumption and transmission, by phone or by current holder in line to the manager of metering data. Always in a logic of interconnected objects, this type of solution can be combined with other sensors (temperature, humidity) in order to provide general and specific information on the buildings forming an intelligent and economic environment.

\subsection{Logistics and Supply chain Management}

Implementation of the internet of things in logistics and presents the many benefit supply chain: elements equipped with RFID and smart shelves that follow the elements in real time, [36] can optimize the many applications, such as: automatically verifies the receipt of goods, followed by stocks, followed out-of-stocks or the detection of shoplifting in real-time traceability in real time using RFID chip fitted equipment, exchange of product data, intelligent management of stocks, automatic checking of the inputs/outputs of products.... These applications will generate a gain of time and significant savings in logistics. Below a summary table of the areas of possible applications of the internet of things.

Table 1. Futuristic application domain.

\begin{tabular}{lll}
\hline Application domain & Description & Example \\
\hline Health & Pharmaceutical products and monitoring & Control pharmaceutical and monitoring of patients. \\
Industry & Commercial or financial activities between the companies & Manufacturing, logistics and services of banks \\
Environments & Protection, monitoring and development of natural resources & $\begin{array}{l}\text { Agriculture, livestock, recycling and management of the } \\
\text { environment }\end{array}$ \\
Society & Development of cities for citizens & Smart house and Smart cities \\
\hline
\end{tabular}

\subsection{The Automotive Field}

The next few years, the communication of vehicle data will be changed gradually from the embedded electronic systems to the software of the wireless sensor network. Nissan Motor Company (Japan) runs on the network of the car in the web2.0 + telematics to achieve automotive architecture. VIP automobiles are fixed with the acceleration of the vehicle axis sensors, alcohol sensors, smoke sensors can be sent to the monitoring office by the general packet radio service (GPRS) in real-time. There are also other applications as the diagnosis of vehicles in real time can be controlled by specific sensors: the tire pressure, fuel consumption and distance between vehicles. All data detected is then rendered to the central system.

\subsection{Field of Robotics}

In future cities, robot taxis swarm together, moving in flocks, providing the service where it is needed in the timely and efficient way. Robot taxis are calibrated to reduce congestion at the bottlenecks in the city and pickup service areas that are more frequently used. A robot is a perfect example of intelligent physical devices. It is usually a system, which, by its appearance, gives the impression that it has intent or a clean body [37].

In closing, we anticipate that research on the IoT technology will continue to evolve over the next decade. 
Several applications of internet of things technology such as new communication technology and information processing and application in the field of robotics and aviation may become available. New approaches and models in the field of logistics for example automatic verification of receipt of the goods, followed stocks, monitoring out-of-stocks or the detection of flight may become available through the use of use of IoT technology.

\section{Conclusion}

The internet of things gives the possibility of merging seamlessly the real world and the virtual world. Thanks to the huge deployment of embedded systems, in fact, the entire range of design for IOT systems options is quite wide, across the open and standardized protocols is much smaller.

The internet of things became a focus for research and development in the last 15 years. A large amount of investments for internet of things was and is still being made by government agencies and industry worldwide.

As described in this document, the internet of things gives an idea of the possibilities offered by a number of existing and future technologies which, together, could in the next 510 years, change the mode of functioning of our societies in depth. It is an evolution of our information and communication systems that will result in the internet of things but the acceptance of IoT by the company will be strongly linked to respect for privacy and the protection of personal data we hope that this survey will be useful for researchers and practitioners in the field, helping them to understand the huge potential of IoT and what are the main fields of application of the internet of objects that are capable of transforming the IoT to a vision of research actually.

\section{Future Directions}

According to our Survey of Object Internet Technologies and Applications, much research is needed to make the IdO Paradigm come true. In this section, future research directions are suggested: development of many applications closely or directly applicable to our present life, such as personal and social areas, mobility and transportation, enterprise fields and industry areas.

Security and privacy issues should be considered very seriously since IdO not only handles huge amounts of sensitive data (personal data, business data, etc.), but also has the power to influence the physical environment with its control capabilities. Cyber-physical environments therefore need to be protected from any form of malicious attacks.

Identify, categorize and categorize IdO technologies, devices and services that will drive IdO development and support IdO vision.

Designing architecture standards would have well defined abstract data models, interfaces and protocols, as well as concrete links to neutral technologies in order to support the broadest possible human beings, software, objects or intelligent devices.
Development of new frameworks for global identification programs, identity management, identity / encryption, authentication, and the creation of global directory search and discovery services for 'IdO with various identification schemes.

\section{References}

[1] Coetzee. L, Fksteen. J, "The internet of things - promise for the future? An introduction", IST-Africa Conference Proceedings, 2011.

[2] M. Aazam, I. Khan, A. A. Alsaffar, E.-N. Huh, "Cloud of internet integrating Internet of things and cloud computing and the issues involved", 11th International Conference on Applied Sciences and Technology (IBCAST), 2014.

[3] H. Sundmaeker, P. Guillemin, P. S. Woelfflé, "Vision and Challenges for Realising the Internet od Things", Gerald Santucci, Head of Unit DG INFSO Unit D.4; European Commission, Mars 2010.

[4] P. Jappinen, R. Guameri, L. M. Correia, "An applications perspective onto the Future Internet", Journal of Network and Computer Applications, vol.36, pp: 249-254, 2013.

[5] Palattella M., Accettura N., Vilajosana, X. et al., «Standardized Protocol Stack for the Internet of (Important) Things», IEEE Communications Surveys \& Tutorials, 2013.

[6] D. Miorandi, S. Sicari, F. De Pellegrini, I. Chlamtac, «Internet of things: Vision, applications and research challenges», Ad Hoc Networks, vol.10, pp: 1497-1516, 2012.

[7] Luigi Atzori, Antonio Iera, Giacomo Morabito, "The Internet of Things: A survey", Computer Networks, vol.54, pp. 27872805,2010 .

[8] D. Singh, G. Tripathi, Antonio J. Jara, «A survey of Internetof-Things: Future Vision, Architecture, Challenges and Services», IEEE World Forum on Internet of Things (WFIoT), 2014.

[9] Eleonora Borgia, "The Internet of Things vision: Key features, applications and open issues", Computer Communications, Vol. 54, pp: 1-31, 2014.

[10] R. Khan, S. U. Khan, R. Zaheer and S. Khan, "Future internet: The internet of things architecture, possible applications and key challenges," in Frontiers of Information Technology (FIT), 2012 10th International Conference On, 2012, pp. 257260.

[11] J. Gubbi, R. Buyya, S. Marusic and M. Palaniswami, "Internet of Things (IoT): A vision, architectural elements, and future directions," Future Generation Comput. Syst., vol. 29, pp. 1645-1660, 2013.

[12] P. Lopez, D. Fernandez, A. J. Jara and A. F. Skarmeta, "Survey of internet of things technologies for clinical environments," in Advanced Information Networking and Applications Workshops (WAINA), 2013 27th International Conference On, 2013, pp. 1349-1354.

[13] D. Yang, F. Liu and Y. Liang, "A survey of the internet of things," in Proceedings of the 1st International Conference on E-Business Intelligence (ICEBI2010), 2010, pp. 358-366. 
[14] A. Gluhak, S. Krco, M. Nati, D. Pfisterer, N. Mitton and T. Razafindralambo, "A survey on facilities for experimental internet of things research," Communications Magazine, IEEE, vol. 49, pp. 58-67, 2011.

[15] Z. Sheng, S. Yang, Y. Yu, A. V. Vasilakos, J. A. McCann and K. K. Leung, "A survey on the ietf protocol suite for the internet of things: standards, challenges, and opportunities," Wireless Communications, IEEE, vol. 20, pp. 91-98, 2013.

[16] Y. Huang, Z. Yang, S. Xiong "Design and Implementation of A Bicycle Parking System Based on Internet-of-things" National Conference on Information Technology and Computer Science (2012). Pp: 593 - 596.

[17] A. Bielsa (2012, Feb.10) Smart Water project in Valencia to monitor Water Cycle Management [Online] Available: http://www.libelium.com/smart_water_cycle_monitoring_sens or_network/

[18] Q. Wei, S. Zhu, C. Du, "Study on key technologies of Internet of Things perceiving mine," Procedia Engineering, vol.26, pp.2326-2333, 2011.

[19] B. Karakostas, "A DNS architecture for the Internet of Things: a case study in transport logistics," Procedia Computer Science, vol.19, pp.594-601, 2013.

[20] Y. Zhang, B. Chen, and X. Lu, "Intelligent monitoring system on refrigerator trucks based on the Internet of Things," Wireless Communications and Applications, vol.72, pp.201206, 2012.

[21] Imran Erguler, "A potential weakness in RFID-based Internetof-things systems", pervasive and mobile computer, vol. 20, pp. 115-126, 2015.

[22] H. Yang and S. Ynag "RFID sensor network architectures to integrate RFID, sensor and WSN" Measurement and Control, pp.56-59, 2007.

[23] J. Yick, B. Mukherjee, D. Ghosal, "Wireless sensor network survey”, Computer Networks, Vol. 52, pp. 2292-2330, 2008.

[24] Ghofrane Fersi, "Middleware for internet of things: a study", International Conference on Distributed Computing in Sensor Systems, Vol. 10, pp: 230-235 2015.

[25] Mell. P, Grance. T, "The NIST definition of cloud computing", National Institute of Standards and Technology, vol. 6, pp: 53-50, 2009.
[26] K. Kai, P. Zhi-bo, W. Cong, «Security and privacy mechanism for health internet of things», The Journal of China Universities of Postsand Telecommunications, Vol. 20, pp: 6468, December 2013.

[27] A. K. Evangelos, D. T. Nikolaos, C B. Anthony, "Integrating RFIDs and smart objects into a Unified Internet of Things architecture", Advances in Internet of Things, 2011.

[28] Danilo F. S. Santos, Hyggo O. Almeida, Angelo Perkusich, “A personal connected health system for the Internet of Things based on the Constrained Application Protocol", Computers and Electrical Engineering, vol 15, 2015.

[29] Alexandre Santos, Joaquim Macedo, António Costa, M. João Nicolau, "Internet of Things and Smart Objects for M-Health Monitoring and Control", Procedia Technology, vol.16, pp: 1351-1360, 2014.

[30] Mari Carmen Domingo, "An overview of the Internet of Things for people with disabilities", Journal of Network and Computer Applications, vol. 35 pp: 584-596, 2012.

[31] Hamid Zargari Asl, Antonio Iera, Luigi Atzori, Giacomo Morabito, "Analysis of the properties of a social network of IoT devices based on real data", IEEE Xplore Digital Library, vol. $6,2013$.

[32] Charith Perera, "The Emerging Internet of Things Marketplace From an Industrial Perspective: A Survey", IEEE Transactions on Emerging Topics in Computing, 2015.

[33] Andrea Zanella, Nicola Bui, Angelo Castellani, Lorenzo Vangelista, Michele Zorzi, "Internet of Things for Smart Cities", IEEE INTERNET OF THINGS JOURNAL, vol. 11, 2014.

[34] J. Clerk Maxwell, "A Treatise on Electricity and Magnetism", $3^{\text {rd }}$ ed., vol. 2. Oxford: Clarendon, 1892, pp.68-73.

[35] Farhana Jabeen, Sarfraz Nawaz, "In-network wireless sensor network query processors: State of the art, challenges and future directions", Information Fusion, vol. 25, pp: 1-15, 2015

[36] Yaqi Xu, Rui Jiang, Shiyi Yan, Di Xiong, "The Research of Safety Monitoring System Applied in School Bus Based on the Internet of Things", Procedia Engineering, vol. 15, pp: $2464-246,2011$.

[37] Yinong Chen, Hualiang $\mathrm{Hu}$, "Internet of intelligent things and robot as a service", Simulation Modelling Practice and Theory, vol. 34, pp: 159-171, 2013. 